\title{
Introducción al comportamiento del fuego
}

Recibido: 12 xaneiro 2009 / Aceptado: 3 marzo 2009

(C) IBADER- Universidade de Santiago de Compostela 2009

Resumen En este documento describen, y conectan entre sí, aspectos básicos del comportamiento del fuego en los incendios forestales. También se hace referencia a diversas herramientas de predicción de utilidad para el manejo del fuego.

Palabras clave Combustible . Tiempo atmosférico . Topografía $\cdot$ Humedad

Summary In this document basic fire behaviour aspects are described and interconnected. Also several prediction tools for fire management are refered to.

Keywords Fuel · Weather · Topography · Moisture

\section{Introducción}

Los incendios forestales no son todos iguales, existe gran variabilidad en cuanto a su dificultad de control, su peligrosidad de cara al personal de extinción, y sus implicaciones ecológicas. Los responsables de la extinción unas veces ven que se puede actuar directamente sobre el frente del incendio mientras que en otras ocasiones ni se lo plantean por el riesgo que conlleva, o por la falta de eficacia de la actuación.

Cada incendio "se comporta", es decir, evoluciona o progresa de forma distinta porque se desarrolla en un ambiente diferente. Los cambios, que a lo largo del espacio y del tiempo, se producen en el comportamiento del fuego se deben a las variaciones que se dan en los distintos

Ana Daría Ruiz González

Unidad de Gestión Forestal Sostenible. E.P.S. Universidad de Santiago de Compostela. Campus Universitario s/n., 27002 Lugo. Tfno: 982285900 (ext 23220). Fax: 982285926 e-mail: anadaria.ruiz@usc.es componentes de su entorno de desarrollo. Dicho entorno nunca será exactamente igual pues está formado por numerosos factores que además, en gran medida, están interconectados.

En los siguientes párrafos se pasa revista a los principales factores que configuran el medio de desarrollo del incendio, así como a las variables cuya estimación permite valorar de forma objetiva el nivel de dificultad y de peligrosidad a la hora del control del incendio. También se incluye una clasificación en función de los estratos que intervienen en la propagación del fuego y se hace referencia a diversas herramientas de predicción.

\section{El entorno de desarrollo del incendio}

En la tabla 1 se enumeran, clasificados en cuatro categorías, los principales factores que condicionan el comportamiento de fuego (Pyne et al., 1996). Los combustibles hay que contemplarlos desde dos niveles distintos: partícula individual y complejo. Las partículas son las distintas partes, u órganos, de los vegetales: acículas, hojas, ramas y ramillas de distintos grosores, fragmentos de corteza, frutos. El complejo, también conocido como lecho de combustible, es el conjunto de partículas heterogéneas en cuanto a forma y propiedades fisicoquímicas que en un momento dado constituyen el medio de propagación del fuego (Guijarro, 2003). Las propiedades del complejo son fácilmente modificables y por tanto sobre las que ha de incidir la prevención de incendios. Reduciendo cargas se atenúa la producción de energía y modificando la estructura se dificulta la transmisión de calor, y por tanto la propagación.

\section{Caracterización del comportamiento del fuego}

Su finalidad es valorar de forma objetiva cual es el nivel de dificultad y de peligrosidad de control del incendio, lo cual es de utilidad a la hora de planificar cómo y con qué medios y 


\section{Factores atmosféricos}

* Temperatura y humedad relativa del aire

* Velocidad y dirección del viento

* Días sin llover

* Nubosidad

* Estabilidad/Inestabilidad atmosférica

Propiedades de las partículas individuales

* Térmicas:

* Capacidad de calor

* Poder calorífico

* Conductividad térmica

* Químicas:

* Composición

* Humedad

* Físicas

* Grosor

* densidad

\section{Factores topográficos}

* Pendiente

* Configuración del terreno

* Orientación de la ladera

* Altitud

\section{Propiedades del complejo}

* Cantidad de combustible:

* Por unidad de superficie

* Por unidad de volumen

* Compactación

* Continuidad/discontinuidad:

* Vertical

* Horizontal

* Relación partículas muertas/vivas

* Relación elementos finos/gruesos
Tabla 1.- Factores que constituyen el ambiente de desarrollo del incendio. Su interconexión determina la energía que desprende el incendio y la efectividad de los mecanismos de transmisión de calor. Consecuentemente determinan la dificultad y la peligrosidad de control del fuego recursos actuar. La caracterización se consigue a través de la estimación de una serie de parámetros conocidos como variables básicas o fundamentales: velocidad de propagación, calor por unidad de superficie, intensidad de reacción, intensidad lineal y longitud de llama. Estos parámetros se pueden determinar sobre cualquier punto del perímetro en llamas del incendio. Sin embargo, donde más interés presenta su conocimiento es en el frente ya que es la zona más crítica, donde la dimensión de la llama es mayor y donde la energía se libera más rápidamente.

La velocidad de propagación se define como la distancia de avance del frente medida perpendicularmente al perímetro en llamas y referida a la unidad temporal. Favorecen la velocidad (Chandler et al., 1991): i) el predominio de elementos finos en el complejo pues se secan, e inflaman, antes que los gruesos; ii) la existencia de "huecos"entre las partículas. Los complejos poco compactos, bien ventilados, garantizan una buena transmisión de calor entre sus elementos, un buen aporte de oxígeno, necesario para la combustión, y el secado rápido de los combustibles; iii) niveles bajos de humedad de los combustibles muertos en respuesta a la ausencia de precipitaciones así como a las altas temperaturas y bajas humedades relativas, fundamentalmente en días soleados (Ruiz \& Vega, 2007); iv) humedad baja de las plantas vivas, reflejo de su estado fisiológico, época del año y disponibilidad de agua en los suelos; v) la presencia de compuestos orgánicos volátiles, de bajo punto de inflamación; vi) bajo contenido en minerales, pues intervienen en las reacciones de degradación térmica de la celulosa, favoreciendo la producción de residuos carbonosos y limitando la formación de gases inflamables; vi) el viento y la pendiente, sobre todo si sus direcciones coinciden, y por tanto sus efectos se suman. El viento, además de aportar oxígeno a la zona en combustión, arroja la llama y el flujo de gases calientes hacia los combustibles que están por delante del frente favoreciendo su deshidratación y su inflamación. La pendiente también acorta la distancia entre la llama y los combustibles sin arder.

El calor por unidad de área es el desprendido por el combustible que arde en cada unidad de superficie del complejo cuando el frente de llamas pasa sobre la misma, durante todo el tiempo que permanece la llama en ese lugar. No incluye por tanto la combustión postfrontal (lenta o incandescente), secundaria desde el punto de vista de la peligrosidad de control del incendio, pero de gran importancia a la hora de explicar los efectos del fuego sobre la recuperación de la vegetación y sobre los suelos. La distinta disponibilidad de combustible asociada a cada incendio implica que se den diferencias muy grandes en el calor por unidad de superficie. La disponibilidad varía enormemente entre complejos pero también dentro de cada complejo oscila continuamente pues es un reflejo de la humedad de sus partículas. Los cambios temporales en disponibilidad pueden ser muy rápidos cuando en el complejo predominan las partículas finas y muertas mientras que serán lentos si predominan los vegetales vivos. El poder calorífico de los combustibles que están ardiendo, condicionado por su composición química, y favorecido por la presencia de volátiles (Elvira \& Hernando, 1989), también afecta al calor por unidad de superficie.

La intensidad de reacción y la intensidad lineal son variables indicativas de la potencia del incendio (Andrews \& Rothermel, 1982). Aportan informan sobre la energía desprendida en la fase de llama y sobre su velocidad de liberación. La intensidad de reacción se obtiene dividiendo el calor por unidad de área entre el tiempo que permanece la llama sobre el mismo punto, sin desplazarse. La intensidad lineal es el calor liberado, en la unidad de tiempo, por cada unidad de longitud del frente (superficie de ancho unitario y largo la profundidad de la llama). Favorecen la intensidad lineal los factores que contribuyen a incrementar la velocidad de propagación y los que contribuyen a 
incrementar el calor por unidad de superficie pues es el producto de ambas variables.

Por último, la longitud de llama es la distancia entre su extremo y la superficie (sin contar ráfagas instantáneas y medida según la dirección de la llama). Se relaciona con la intensidad lineal a través de expresiones empíricas del tipo:

$$
L=a I_{L}^{b}
$$

Donde $L$ es la longitud de la llama mientras que a y b son coeficientes positivos que dependen del complejo.

\section{Tipos de incendios}

En la tabla 2 se muestra la clasificación de los incendios por el estrato/os de combustible que intervienen en la propagación del fuego.

En el fuego de superficie la llama se propaga a través de los restos finos en contacto directo con el suelo, o a través de los elementos finos, ya sean vivos o muertos, de las plantas de baja altura: vegetación herbácea, matorral, arbustos o arbolillos (Pyne et al., 1996). El fuego de copas se origina cuando el calor que desprende la fase de superficie, y que se transmite hacia arriba por convención, supera la energía necesaria para lograr la inflamación de los elementos finos de las copas. Sin embargo, aunque las copas se inflamen no está asegurada la propagación a través del dosel a no ser que el flujo de combustible fino hacia la zona en combustión supere un determinado nivel (Van Wagner, 1977; Alexander, 1988). La continuidad vertical entre estratos de combustible favorece la transición del fuego de superficie a las copas. También los valores bajos de humedad foliar, la presencia de compuestos orgánicos volátiles en las hojas, y todos aquellos factores que incrementan la intensidad y la longitud de llama del incendio de superficie. La propagación a través de las copas se facilita con valores bajos de humedad foliar y altos de biomasa foliar por unidad de volumen de copa.

En el fuego de copas pasivo se inflaman árboles individuales, o grupos reducidos de árboles, pero no prospera la propagación del fuego a través del estrato aéreo de forma continua, de ahí que también se les conozca como

\begin{tabular}{ll} 
& \multicolumn{2}{c}{ Tipos de incendios } \\
$* \quad$ Suelo \\
$* \quad$ Superficie \\
$* \quad$ Copas \\
$* \quad$ Pasivo, falso o antorcheo \\
$* \quad$ Activo, integral o continuo \\
$* \quad$ Independiente \\
$* \quad$ Intermitente
\end{tabular}

Tabla 2.- Clasificación de los incendios forestales en función del estrato/os implicados en su propagación falsos incendios de copa. La combustión de las copas está totalmente controlada por la fase superficial. El fuego de copas activo se propaga de forma continua por el estrato aéreo pero sigue necesitando para mantenerse en todo momento del calor generado por el incendio de superficie. Ambos fuegos, el de superficie y el de copas, se propagan simultáneamente, a la misma velocidad y como una pared en llamas bien definida que se extiende de la superficie a las copas (Scott \& Reinthardt, 2001). El fuego de copas independiente se inicia a partir de un incendio de superficie pero posteriormente avanza por su cuenta, empujado por el viento y a mayor velocidad que el de superficie que se queda retrasado. Son fuegos que una vez iniciados dejan de depender de la columna de convección que se eleva desde el fuego de superficie, el calor necesario para la propagación del fuego por las copas lo proporciona su propia combustión. Ocurren en raras ocasiones y son de corta duración (Van Wagner, 1993), precisan de determinadas combinaciones de pendiente, elevada velocidad del viento y baja humedad foliar.

El Canadian Forest Fire Behavior Prediction System (Forestry Canada Fire Danger Group, 1992) utiliza el término de fuego intermitente para describir un incendio que alterna, en el espacio y en el tiempo, entre fuego de copas continuo y fuego de superficie, o fuego de copas pasivo. Es un fenómeno que se puede dar cuando hay variabilidad espacial en los combustibles tanto de superficie como de copas o cuando hay variabilidad temporal en la velocidad del viento.

En comparación con los incendios de superficie (Scott \& \& Reinthardt, 2001), y desde el punto de vista de la dificultad y peligrosidad de control, los incendios de copas: i) son más rápidos e intensos, impiden acercarse al incendio; ii) generan mayor cantidad de pavesas volantes, y además de mayor alcance, incrementando el riesgo para el personal de extinción; iii) presentan mayor riesgo para las personas ajenas a la extinción, y para sus bienes, cuando se desarrollan en la cercanía de zonas residenciales o recreativas.

Por último, los fuegos de suelo se propagan mediante combustión sin llama a través del mantillo, raíces o restos leñosos enterrados y parcialmente descompuestos. Avanzan mucho más lentamente que los anteriores pero de forma continua y persistente, incluso con altos contenidos en humedad de los combustibles. También se originan a partir de un fuego de superficie y mantienen en todo momento la capacidad potencial de aflorar de nuevo a la superficie y reactivar la formación de las llamas. Provocan graves daños a los órganos subterráneos de las plantas.

\section{Herramientas de planificación y gestión}

La estimación de la velocidad de propagación, o de la intensidad lineal, en las diferentes formaciones vegetales, bajo determinadas condiciones topográficas y atmosféricas, proporciona información objetiva de gran utilidad tanto para la prevención de incendios como para la planificación de la extinción. 
El poder pronosticar las situaciones para las que una determinada masa arbolada puede llegar a desarrollar fuego de copas, o el poder comparar la potencialidad de diversas masas frente a dichos fuegos es fundamental a la hora de diseñar, y priorizar, tratamientos selvícolas y de control del combustible superficial.

En la actualidad existen modelos que nos permiten efectuar pronósticos sobre el comportamiento del fuego y sobre el tipo de incendio a desarrollar bajo diferentes condiciones del medio. Los principales modelos, o al menos los más difundidos internacionalmente, se encuentran implementados en programas informáticos conocidos como Sistemas de Simulación de Incendios. Entre ellos destacan BehavePlus fire modeling system (Andrews et al., 2005), FARSITE: Fire Area Simulator (Finney, 1998), NEXUS (Scott, 1999) y FFE-FVS (Fire and Fuels Extension to de Forest Vegetation Simulator) (Reinhardt \& Crookston, 2003) del USDA Forest Service y disponibles, en su mayor parte, en http://www.fire.org. Todos ellos se basan en la internacionalmente reconocida ecuación de propagación de Rothermel (1972) para la predicción de la velocidad de propagación del fuego de superficie y tienen un claro antecesor, el sistema BEHAVE (Fire Behavior Prediction and Fuel Modeling System) (Andrews, 1986; Andrews \& Chase, 1989) que data de los años 80 , y que fue durante muchos años el único software disponible para el manejo del fuego.

Los modelos de predicción del comportamiento del fuego suponen una herramienta muy importante en la planificación y gestión de incendios, y más aún cuando se encuentran implementados en sistemas de simulación de fácil manejo como son los anteriormente mencionados. Sin embargo, es de suma importancia recordar que su uso puede complementar, pero nunca suplir, a la experiencia adquirida en campo ante situaciones reales. Tampoco se deben de utilizar los modelos si no se tienen los conocimientos básicos para entenderlos, y analizar los resultados. Por otro lado, hay que recordar que pueden existir otros modelos quizá menos conocidos pero más cercanos, y específicos para determinados complejos y condiciones atmosféricas. Por ejemplo, en el caso de Galicia, los del Centro de Investigación e Información Ambiental de Lourizan para la propagación de fuego a través de las formaciones de matorral habituales en la Comunidad (Vega et al.,1998; 2006).

\section{Bibliografía}

Alexander, M.E. (1988). Help with making crown fire hazard assessments. In: Fischer, W. C.; Arno, S.F., comps. Protecting people and homes from wildfire in the Interior West: proceedings of the symposium and workshop. 1988 October 6-8, Missoula, MT.

Andrews, P.L. (1986). BEHAVE: Fire Behavior Prediction and Fuel Modeling System-BURN Subsystem, Part 1. USDA Forest Service. Gen. Tech. Rep. INT-194.
Andrews, P.L \& CHASE, C.H. (1989). BEHAVE: Fire Behavior Prediction and Fuel Modeling System-BURN Subsystem, Part 2. USDA Forest Service. Gen. Tech. Rep. INT-260.

Andrews, P.L. \& Rothermel, R.C. (1982). Charts for interpreting wildland fire behaviour characteristics. USDA For. Ser. Gen. Tech. Rep. INT-131.

Andrews, P.L., Bevins, C.D. \& Seli, R.C. (2005). BehavePlus fire modelling system Version 3.0. User's Guide. USDA forest Service. Gen. Tech. Rep. RMRS-GTR- 106WWW Revised, Ogden, UT.

Chandler, C., Cheney, P., Thomas, P., Trabaud, L. \& Williams, D. (1991). Fire in forestry. Volume I: Forest fire behavior and effects. Krieger Publishing Company, Malabar, Florida.

Elvira, L.M. \& Hernado, C. (1989). Inflamabilidad y energía de las especies de sotobosque. MAPA. Monografía INIA, Madrid.

Finney, M.A. (1998). FARSITE: Fire area simulator-model development and evaluation. USDA Forest Service. Res. Pap. RMRS-RP-4. Ogden, UT.

Forestry Canada Fire Danger Group. (1992). Development and structure of the Canadian Forest Fire Behavior Prediction System. For. Can. Sci. and Sust. Dev. Inf. Rep. ST-X-3.

Guijarro, M. (2003). Comportamiento del fuego y régimen térmico en diferentes complejos de combustible forestal. Ministerio de Ciencia y Tecnología, Tesis doctoral INIA, Madrid.

Pyne, S.J., Andrews, P.L. \& Laven, R.D. (1996). Introduction to wildland fire. John Wiley \& Sons, Inc, USA.

Reinhardt, E.D., Crookston, N.L. (2003). The Fire and Fuels Extension to de Forest Vegetation Simulator. USDA Forest Service. Gen. Tech. Rep RMRS-GTR-116. Odgen, UT.

Rothermel, R.C. (1972). A mathematical model for predicting fire spread in wildland fuels. USDA Forest Service. Intermountain Forest and Range Experiment Station. Res. Pap. INT-115.

Ruiz A.D, Vega J.A. (2007). Modelos de predicción de la humedad de los combustibles muertos: Fundamentos y aplicación. Ministerio de Educación y Ciencia, Instituto Nacional de Investigación Agraria y Alimentaria. Monografías INIA: Serie Forestal Número 15, Madrid.

Scott, J.H. (1999). NEXUS: A system for assessing crown fire hazard. Fire Management Notes 59(2):20-24.

Scott, J.H. \& Reinhardt, E.D. 2001. Assesing crown fire potencial by linking models of surface and crown fire behavior. USDA Forest Service. Res. Pap. RMRS-RP-29. Rocky Mountain Research Station.

Van Wagner, C.E. (1977). Conditions for the start and spread of crown fire. Can. J. For. Res., 7: 23-24. 
Van Wagner, C.E. 1993. Prediction of crown fire behavior in two stands of jack pine. Canadian Journal of Forest Research. 23: 442-449.

Vega, J.A., Cuiñas, P., Fortúrbel, T., Pérez-Gorostiaga, P. \& Fernández, C. (1998). Predicting fire behaviour in Galician (NW Spain) shrubland fuel complexes. In: Viegas, D.X. (Ed.). Proc. 3rd Int. Conf. on Forest Fire Research/14th Fire and Forest Meteorology Conf. Luso. 16-20 November 1998. 713-728.
Vega, J.A., Fernandes, P., Cuiñas, P., Fontúrbel, M.T., Pérez, J.R. \& Loureiro, C. (2006). Fire spread analysis of early summer field experiments in shrubland fuel types of northwestern Iberia. In: Viegas, D.X. (Ed.) Proc. 5th Int. Conf. on Forest Fire Research. Figueira da Foz 27-30 November 2006. Elsevier, Amsterdam. 